\title{
Dificultades de las Familias para Participar en los Centros Escolares
}

\section{Difficulties of Families to Participate in Schools}

\author{
Beatriz Rodríguez-Ruiz ${ }^{1 *}$ \\ Raquel-Amaya Martínez-González ${ }^{1}$ \\ $\mathrm{M}^{\mathrm{a}}$ José Rodrigo López ${ }^{2}$ \\ ${ }^{1}$ Universidad de Oviedo ${ }^{2}$ Universidad de La Laguna
}

\begin{abstract}
La participación de las familias en los centros escolares beneficia al alumnado, a las familias y a la institución escolar. Sin embargo, existen condicionantes que dificultan esta participación y que conviene analizar para sugerir estrategias de actuación. Este es el objetivo de este estudio, en el que han participado 880 padres y madres españoles con hijos escolarizados en $2^{\circ}$ y $4^{\circ}$ de la enseñanza secundaria obligatoria cumplimentando la Escala de Relaciones de Confianza entre la Familia y el Centro Escolar (adaptado por Martínez-González, 1996). Los resultados obtenidos tras realizar contrastes de grupos considerando el género, edad, nivel de estudios y situación laboral de los progenitores indican que las mayores dificultades de participación se deben al horario laboral (padres) y a la crianza de los hijos (madres). Se requieren estrategias de conciliación de la vida laboral y familiar, así como adecuar los horarios de los centros para posibilitar la participación familiar.
\end{abstract}

Descriptores: Familia, Centro escolar, Participación, Dificultades.

Parental involvement in schools is positive for the students, their families and the schools themselves. However, there are circumstances that make it difficult for parents to participate, and which deserve attention in order to promote intervention measures to this regard. This is the objective of this study, in which 880 Spanish fathers and mothers with children enrolled in the 2nd and 4th grade of compulsory secondary school filled in the Family-School Relationship Trust Scale (adapted by Martínez-González, 1996). The results obtained after performing group comparisons taking into account the gender, age, level of literacy and work circumstances of the parents, show that the main difficulties to participate come from work timetable (fathers) and children's upbringing (mothers). Thus, work and family life conciliation measures are needed as well as adapting the school timetable to the parents' possibilities to participate.

Keywords: Family, School, Partnership, Difficulties. 


\section{Introducción}

Los continuos cambios que experimentan las sociedades occidentales, que se expresan, entre otros, en indicadores como la incorporación de la mujer al mercado laboral, el descenso de la natalidad, los cambios de estructura, de composición y de dinámicas de las familias o los avances de la sociedad de la información y la comunicación y su influencia en la ciudadanía, ponen de manifiesto la necesidad de promover nuevas líneas en la acción educativa. Entre ellas se plantea la conveniencia de reforzar la colaboración entre las familias y los centros escolares (Martínez González, Rodríguez y Jimeno, 2010).

Las investigaciones realizadas sobre esta temática (Castelli, Mendel y Ravn, 2003; Castelli, Addimando, Pieri y Pepe, 2011; Hiatt-Michael, 2001; Martínez González, Pérez Herrero y Rodríguez Ruiz, 2005; Phtiaka y Symeonidou, 2007) indican que existen diferentes puntos de vista sobre cuál es el papel que desempeñan las familias y los centros escolares y sobre el tipo de colaboración que se puede establecer entre ambas entidades.

Tradicionalmente se ha entendido que desde el ámbito escolar y familiar la acción educativa se focaliza en objetivos diferentes, de modo que mientras la familia cubre las necesidades básicas de los niños ${ }^{1} \mathrm{y}$ adolescentes, principalmente afectivas, el profesorado asume su formación académica (Rivera y Milicic, 2006; Smit y Driessen, 2005). Sin embargo, investigadores como Epstein y Sanders (2006) sostienen que los padres y las madres han de ocuparse también de preparar a sus hijos para asistir al centro escolar, guiar su aprendizaje y crear un clima que favorezca su desarrollo integral y su adaptación al ámbito escolar.

Diversos autores (Comisión Europea, 2000; Epstein, 2011; Glasgow y Whitney, 2009; San Fabián, 1994) han señalado que la participación de los padres y madres en los centros escolares se justifica, entre otros motivos, porque permite aproximar las culturas familiar y escolar, favorece la educación de los niños y su rendimiento escolar y constituye, de esta manera, un indicador de calidad educativa.

Las experiencias que los niños y los adolescentes viven en la familia y en el centro escolar han de gozar de cierta continuidad procurando que ambos contextos se apoyen mutuamente (García Bacete y Traver, 2010). Por otro lado, la situación de minoría de edad del alumnado en los centros escolares subraya la necesidad de implicar a las figuras adultas del contexto familiar (Martín y Gairín, 2007).

No obstante, los padres y madres suelen presentar visiones diferentes de su colaboración con el centro escolar en función de la etapa en la que se encuentren sus hijos; en general, perciben que es más fácil colaborar en las etapas de Educación Infantil y Primaria que en la etapa de la Educación Secundaria Obligatoria (Deslandes y Bertrand, 2005). Ello se debe, en parte, a que consideran que en los primeros años de escolarización de sus hijos es más necesaria su implicación y a que se sienten más competentes para apoyarles en ese momento (Rivera y Milicic, 2006). En la etapa de la educación secundaria obligatoria los informes académicos existentes sitúan a los estudiantes españoles en una delicada situación que se caracteriza por un alto porcentaje de fracaso escolar, de bajo rendimiento académico y altos índices de absentismo (Broc, 2006; Calero, Choi y Waisgrais, 2010; Fernández, Mena y Riviere, 2010; Lozano, 2003; Martínez González y Álvarez Blanco,

\footnotetext{
${ }^{1}$ Los términos genéricos niño(s), hijo(s) hacen referencia tanto al género masculino como al femenino.
} 
2005). Estos aspectos preocupan no sólo al profesorado sino también a las familias, que intentan implicarse activamente en los estudios de sus hijos, a pesar de muchos condicionantes. Por ello, parece necesario explorar la relación y colaboración entre el contexto familiar y escolar desde la perspectiva de las familias, tomando en consideración las dificultades que limitan su participación en los centros, y que será el objetivo de esta publicación.

Desde una aproximación normativa y legislativa se contempla el derecho de los padres a participar con los centros en la educación de sus hijos; esta idea está recogida en la Declaración Universal de los Derechos Humanos, así como en la Constitución Española y en las Leyes de Educación que se han ido sucediendo hasta el momento actual. En ellas se plantea el derecho a participar en la toma de decisiones en los centros por parte de todos los agentes que componen la comunidad educativa. En muchos centros se desea trabajar juntos para conseguir metas socializadoras y educativas adecuadas para los niños y adolescentes (Martínez González et al., 2010; Ministerio de Educación Cultura y Deporte, 2014).

Ello requiere compartir información, llegar a acuerdos mutuos sobre los roles a desempeñar, asumir responsabilidades conjuntas y mantener relaciones basadas en el respeto, la lealtad, la confianza y la honestidad. En este sentido, existe cierto consenso en utilizar el término partnership para referirse a la acción conjunta y compartida entre el centro escolar, las familias y también la comunidad, como un proceso en el que todos los agentes implicados se apoyan mutuamente e intentan armonizar sus contribuciones para mejorar el aprendizaje de los alumnos, su motivación y su rendimiento escolar (Epstein, 2011; Martínez González et al., 2005; Ravn, 2003). De este modo, esta colaboración se refiere no solo al contexto familiar y escolar, sino que asumiendo la perspectiva ecológica del desarrollo humano (Bronfenbrenner, 1979), incorpora también las aportaciones de los entornos comunitarios, de las entidades educativas no formales, medios de comunicación, los grupos sociales, etc., con los que las familias y los centros escolares también interactúan cotidianamente (Davis, 1991).

Como entidad educativa formal, se entiende que el centro escolar ha de desempeñar un papel fundamental en la promoción de esta colaboración. Por ello se anima al profesorado a invitar y motivar a los padres y madres a participar en el centro, a valorar las potencialidades de las familias y de su trabajo conjunto, y a centrarse en aquellos aspectos que son percibidos como fundamentales y necesarios para un correcto desarrollo de la función educativa y socializadora (García Bacete, 1996). En esta línea, Anderson y Minke (2007) consideran que la invitación directa del profesorado a las familias para que participen en el centro escolar es una de las variables que más influyen en la decisión que tomen éstas para participar. No obstante, la institución escolar se ha caracterizado por dar más importancia a los aspectos académicos y curriculares que a las relaciones interpersonales con las familias en el proceso educativo. En algunos estudios se muestra incluso que estas relaciones pueden llegar a ser tensas y conflictivas (Pepe y Addimando, 2010; Van der Wolf y Everaert, 2005). Esto se hace más evidente en la etapa de la educación secundaria, como consecuencia de una mayor autonomía del alumnado en la gestión de los estudios, un distanciamiento entre la familia y el profesorado como consecuencia del bajo rendimiento escolar o una menor implicación activa de la familia en el curriculum escolar, etc., como ya se ha mencionado anteriormente (Gimeno, 1997). 
De lo comentado se deduce que una buena relación entre los centros escolares y las familias no siempre es fácil de llevar a cabo. Los factores que la dificultan están asociados, como cabe esperar, a todos los agentes implicados: padres y madres, profesorado, alumnado e institución escolar, como los más fundamentales (Christenson, Rounds y Franklin, 1992). Es frecuente que cada uno de ellos, de modo diferente y en diversos aspectos, manifieste temores e incertidumbres que, en algunas ocasiones, llegan a impedir esa colaboración, generando una situación de aislamiento contextual que no favorece a ninguno de los implicados, especialmente al alumnado (Rodríguez-Ruiz, Rodrigo-López y Martínez-González, 2015).

Las variables sociodemográficas de las familias como edad de los padres y madres, nivel educativo de éstos, situación laboral, estatus socioeconómico, número de hijos, tipología familiar o el curso escolar en que se encuentran los hijos, influyen y en algunas ocasiones determinan, la colaboración de la familia con el centro escolar (Garreta, 2008; Jaeggi, Osiek, y Favre, 2003). En este sentido, Berends (1995) encontró que las familias con mayores niveles socioeconómicos y culturales mostraban mayor frecuencia de participación en el centro, lo que repercutía en una mayor motivación escolar en sus hijos y en la cantidad de tiempo que dedican con ellos a realizar tareas escolares. Hart y Risely (1995), en la misma línea, sostienen que los factores de pobreza, diversidad cultural y lingüística de la familia se asocian con una menor implicación de los padres en el centro escolar.

Desde otros estudios se sostiene que la organización horaria del sistema laboral y escolar no favorece la relación y cooperación entre la familia y el centro escolar (Domínguez Martínez, 2010), y que la dificultad para conciliar los horarios familiares y escolares (Maganto, Etxeberria, y Porcel, 2010), más aún cuando los padres y madres trabajan fuera del hogar familiar, constituye el principal obstáculo para la colaboración entre ambos contextos (Martínez-González, Rodríguez-Ruiz y Rodrigo-López, 2012). Así pues, es preciso desarrollar más estudios que permitan identificar con más detalle las circunstancias que dificultan su participación. En esta línea, la investigación que se presenta tiene como objetivo analizar dificultades que encuentran los padres y madres para participar en el centro escolar de sus hijos y su relación con características sociodemográficas como el género de los progenitores, su nivel de estudios, edad y situación laboral. Todo ello con la finalidad de identificar indicadores de diversidad familiar que permitan orientar líneas de actuación diferencial para promover la participación de las familias en los centros escolares.

\section{Método}

\subsection{Participantes}

En el estudio han participado 880 familias españolas con hijos en edad adolescente escolarizados en $2^{\circ}$ y $4^{\circ}$ curso de la Enseñanza Secundaria Obligatoria (ESO) en España. Proceden de dos provincias geográficamente alejadas entre sí y con características socioculturales y económicas diferentes (tasa de natalidad, gasto en educación por habitante, etc.) (Pérez García, 2015), para garantizar cierta representatividad de la diversidad familiar en función de factores geográficos: una se encuentra en la costa septentrional del país-Asturias y otra en las Islas Canarias-Santa Cruz de Tenerife, cerca del continente africano. 
La elección de la etapa de la educación secundaria obligatoria se realizó teniendo en cuenta resultados de investigaciones previas (Ceballos et al., 2012; Deslandes y Bertrand, 2005; Jares, 2006; Martínez González y Álvarez Blanco, 2005; Oliva y Parra, 2004; Rodrigo et al., 2008) que indican una mayor dificultad para establecer relaciones de colaboración entre los centros escolares y las familias en esta etapa que en las de educación infantil y primaria. Se eligió el segundo y cuarto curso de esta etapa escolar por considerar que en ellos los padres y madres tienen ya un conocimiento del centro y experiencia en factores que pueden condicionar su participación.

El perfil socio-demográfico obtenido de las familias participantes con relación a las variables que se analizan en este estudio es como sigue (tabla 1): los progenitores de ambos géneros están representados con el mismo porcentaje del 50\% de padres y 50\% de madres. Prácticamente todos están casados (92\%), con edades que se distribuyen por intervalos de la siguiente manera: $23.4 \%$ entre $30-40$ años, $67.8 \%$ entre 41 y 51 años y $8.8 \%$ entre 52 y 62 años. Respecto al nivel educativo, el 39.8\% de los padres y madres tienen un nivel de estudios secundario (Bachillerato y Formación Profesional), un 30.2\% nivel educativo superior (Universitarios) y otro 30\% nivel educativo primario (Estudios Primarios y Graduado Escolar). Con relación a su situación laboral los datos indican que el porcentaje más alto se sitúa en la categoría de «ocupado/a» con un 80\%; un $13 \%$ señala que trabaja de manera exclusiva y no remunerada en el hogar familiar, siendo todos los casos de género femenino; un 3.6\% son parados y un 3.4\% jubilados. Respecto al número de hijos, el $50.8 \%$ tiene dos, situación que se ajusta con la tendencia nacional de las familias españolas con hijos en edad adolescente (INE, 2014), seguido de un hijo (31.1\%) y de tres o más (18.2\%).

Tabla 1. Distribución de los participantes según variables sociodemográficas

\begin{tabular}{|c|c|c|c|c|}
\hline & $\begin{array}{c}\text { PADRE } \\
\text { M (DT) o \% }\end{array}$ & $\begin{array}{c}\text { MADRE } \\
\text { M(DT) O \% }\end{array}$ & & $\begin{array}{c}\text { TOTAL } \\
\%\end{array}$ \\
\hline Edad & $45.51(5.47)$ & $43.29(5,22)$ & Número de hijos & \\
\hline Estado civil & & & - Uno & $31.1 \%$ \\
\hline - Casado/a & $92.7 \%$ & $92 \%$ & - Dos & $50.7 \%$ \\
\hline $\begin{array}{l}\text { - Divorciado/a- } \\
\text { Separado/a }\end{array}$ & $5.9 \%$ & $6.1 \%$ & - Tres o más & $18.2 \%$ \\
\hline - Soltero/a & $0.5 \%$ & $0.9 \%$ & Provincia & \\
\hline - Pareja de hecho & $0.9 \%$ & $0.9 \%$ & - Asturias & $57.7 \%$ \\
\hline Nivel de estudios & & & - Santa Cruz de Tenerife & $42.3 \%$ \\
\hline $\begin{array}{l}\text { - Bajo: graduado } \\
\text { escolar }\end{array}$ & $32.5 \%$ & $27 \%$ & Zona geográfica & \\
\hline - Medio: Bach. y FP & $38.9 \%$ & $40.7 \%$ & - Rural & $54.8 \%$ \\
\hline - Alto: universitarios & $26.8 \%$ & $31.8 \%$ & - Urbana & $45.2 \%$ \\
\hline Situación laboral & & & Tipo de centro & \\
\hline - Ocupado/a & $92.5 \%$ & $67.5 \%$ & - Público & $39.1 \%$ \\
\hline - Parado/a & $2.3 \%$ & $5 \%$ & - Privado & $22.3 \%$ \\
\hline - Jubilad/a-Pension. & $5.2 \%$ & $1.6 \%$ & - Concertado & $38.6 \%$ \\
\hline - Ama de casa & $0 \%$ & $25.9 \%$ & & \\
\hline
\end{tabular}

Fuente: Elaboración propia.

El porcentaje de respuesta en las dos provincias resultó comparable: 57.7\% en Asturias (6 centros escolares distribuidos según su titularidad de la siguiente manera: tres centros públicos, dos centros concertados y un centro privado) y $45.3 \%$ en la isla de Tenerife (Canarias) (4 centros escolares, de los cuales tres son públicos y uno es privado), así como su distribución en zona urbana (45.2\%) y rural (54.8\%). La tipología de los centros 
participantes resultó en un porcentaje similar entre públicos $(39.1 \%)$ y concertados $(38.6 \%)$. Los centros privados presentan un volumen más bajo (22.3\%) que se corresponde con el también menor número de centros de esta tipología en ambas provincias.

\subsection{Instrumento de recogida de información y variables de estudio}

La recogida de información sobre los aspectos de estudio se ha llevado a cabo con la Escala de Relaciones de Confianza entre la Familia y el Centro Escolar en su versión para padres y madres. Esta escala ha sido adaptada por Martínez González (1996) al castellano del original Family-School Relationship Trust Scale, del Partneship for School Success Program (USA), elaborada originalmente por Sinclair, Lam, Christenson y Evelo (1993). Posteriormente las autoras originales la han referenciado en Adams y Christenson (1998, 2000).

La Escala de la adaptación española presenta una primera parte de datos sociodemográficos, y una segunda parte compuesta por 58 ítems sobre relaciones entre la Familia y el Centro Escolar, clasificados en dos secciones: 1) en una se valora la frecuencia de contactos entre las familias y el profesorado mediante una escala Likert de cinco alternativas (1-Nunca; 2-Pocas veces; 3-Algunas veces; 4-A menudo y 5-Siempre); 2) en otra se analizan las actitudes y percepciones de las familias respecto al centro escolar y al profesorado también mediante una escala Likert de cinco alternativas que expresan grado de acuerdo con las temáticas formuladas (1-Nada de acuerdo; 2-Poco de acuerdo; 3Moderadamente de acuerdo; 4-Muy de acuerdo y 5-Totalmente de acuerdo). Según los planteamientos expresados por Hernández, Muñiz y García (2000) sobre los ítems y sus escalas de medida, en este estudio se ha asumido que las variables latentes a las que se asocian los ítems analizados son continuas categorizadas en cinco niveles de respuesta, lo que permite realizar sobre ellas análisis estadísticos propios de las escalas de intervalo.

$\mathrm{El}$ análisis factorial realizado con el método de ejes principales y rotación oblimin directo (Kayser-Meyer-Olsen=.875, prueba de esfericidad de Bartlett $\chi\left({ }_{1596}\right)=12405.766 ; \mathrm{p}=.000$ ) permitió obtener una estructura factorial con cinco factores que explican el $53.56 \%$ de la varianza, y unos valores de consistencia interna calculados con el Alpha de Cronbach adecuados para cada factor (Cronbach, 1951). Estos cinco factores son: 1) Percepción de las familias sobre la colaboración del profesorado $(\alpha=.93)$; 2) Iniciativa en promover contactos entre la familia y el profesorado $(\alpha=.83) ; 3)$ Implicación de los padres y madres $\mathrm{y}$ actitud del hijo ante el estudio $(\alpha=.70), 4$ ) Apoyo emocional a los hijos ante dificultades académicas y Dificultades para participar en el centro escolar $(\alpha=.67)$, y 5 ) Apoyo familiar ante problemas de estudio de los hijos y participación de los padres en el centro escolar $(\alpha=.71)$.

En este artículo se analizan específicamente los once ítems referidos al Factor 4, clasificados en dos secciones: 1) Apoyo emocional a los hijos ante dificultades académicas (tres ítems) (ejemplo: "Cuando mi hijo me transmite problemas con el profesorado, le muestro mi apoyo incondicional y critico al centro”) y 2) Dificultades de los padres y madres para participar en el centro escolar (ocho ítems) (ejemplo; "Las dificultades que tengo para participar en el centro escolar se deben a que me siento a disgusto en el centro"). 


\subsection{Procedimiento}

Para recoger la información se contactó por carta con 14 centros escolares de las provincias mencionadas, tanto públicos como privados y concertados, y tanto de áreas urbanas como rurales, con la finalidad de disponer de una amplia red de centros con situaciones variadas que puedan reflejar, a su vez, variabilidad en las situaciones familiares.

Cuando los centros escolares consintieron en participar se envió a todas las familias del alumnado de $2^{\circ}$ y $4^{\circ}$ de la Educación Secundaria Obligatoria una carta del equipo investigador en la que se solicitaba su participación, así como cumplimentar un protocolo a través del cual daban su consentimiento, garantizando el equipo de investigación el anonimato de la información suministrada. El procedimiento fue aprobado por el Comité de Ética de la Investigación y de Bienestar Animal (CEIBA) de la Universidad de La Laguna. Se obtuvieron autorizaciones de las familias en un 95\% de los casos, si bien no todas ellas cubrieron finalmente la escala a aplicar. El porcentaje final recogido resultó del $85 \%$. La escala se entregó al alumnado dentro de un sobre que incluía instrucciones muy detalladas sobre cómo cumplimentarla.

\subsection{Análisis de datos}

Los datos obtenidos han sido procesados con el paquete estadístico SPSS.20 a través de: 1) estadísticos descriptivos: frecuencias, porcentajes, medidas de tendencia central (Media, M) y variabilidad (desviación típica, DT); 2) Contrastes de dos grupos para muestras independientes a través de la t de Student, entre las variables analizadas y la variable socio-demográfica género del progenitor (padre y madre). En cada análisis se tuvo en cuenta si se cumplía la condición de homogeneidad de las varianzas (prueba de Levene). Se ha considerado la existencia de diferencias estadísticamente significativas a partir de una probabilidad $\mathrm{p} \leq .05$. Se ha calculado también el tamaño del efecto con la $\mathrm{d}$ de Cohen (1969) cuando las diferencias resultaron significativas; 3) ANOVA de un factor con las variables de análisis y las variables socio-demográficas: edad (30-40 años, 41-51 años y 52-62 años), nivel de estudios (primarios, secundarios y universitarios), situación laboral (ocupado, parado, jubilado y solo labores domésticas) y número de hijos (uno, dos y tres o más). En cada análisis se tuvo en cuenta si se cumplía la condición de homogeneidad de la varianzas (prueba de Levene). Cuando se cumplía esta condición y cuando las diferencias resultaron significativas se realizaron contrastes a posteriori de dos grupos utilizando el método de Scheffé. En los casos en los que no se cumplía la condición de homogeneidad de varianzas se recurrió al estadístico de Welch, como una alternativa robusta. Seguidamente, se realizó un análisis de las diferencias significativas entre los grupos utilizando el método de Games-Howell. En estos últimos se utilizó el estadístico R² como indicador del tamaño del efecto del contraste. Siguiendo las recomendaciones de Cohen (1969) la relevancia clínica de los resultados se clasifica como despreciable cuando $\mathrm{R}^{2}<.01$, baja cuando $\mathrm{R}^{2}>.01$ y $\mathrm{R}^{2}<.09$, media cuando $\mathrm{R}^{2}>.09$ y $\mathrm{R}^{2}<.25$ y alta cuando $\mathrm{R}^{2}>.25$.

Se han realizado análisis comparativos con estas cuatro variables sociodemográficas (género, nivel educativo, edad y situación laboral de los progenitores) por estar consideradas en la literatura científica revisada como factores que pueden incidir de manera diferencial en las dificultades que encuentran las familias para participar en el centro escolar y para actuar ante las dificultades de sus hijos, que constituye el objetivo de este estudio (Garreta, 2008; Jaeggi, Osiek y Favre, 2003). 


\section{Resultados}

La exposición de resultados se realiza en dos secciones. En la primera se presentan indicadores sobre las dificultades analizadas en base a estadísticos descriptivos de tendencia central y variabilidad, y de porcentajes. En la segunda se realizan análisis comparativos entre grupos para identificar diferencias estadísticamente significativas en estos indicadores en función de las variables socio-demográficas consideradas.

\subsection{Indicadores basados en estadísticos descriptivos}

Los análisis descriptivos realizados con cómputo de frecuencias y porcentajes y medidas de tendencia central y variabilidad (tabla 2) indican que, en términos generales, los progenitores que han participado en este estudio no tienen dificultades importantes para participar en el centro escolar. Los valores de las medias aritméticas se sitúan en las opciones Nunca o Pocas veces en dificultades relacionadas con "sentirse a disgusto en el centro escolar" ( $\mathrm{M}=1.42$, DT=0.90), "tener experiencias negativas en el centro escolar" $(\mathrm{M}=1.42, \mathrm{DT}=.92)$ y el "trasporte hasta el centro escolar" $(\mathrm{M}=1.54, \mathrm{DT}=1.02)$. La misma tendencia de respuesta se encuentra en la conducta parental "justifico el comportamiento de mi hijo cuando no cumple las normas de convivencia del centro" $(\mathrm{M}=1.56$, DT $=0.95)$.

A pesar de estos indicadores positivos que reflejan la no existencia de dificultades en la mayoría de las familias consultadas, los resultados muestran también la tendencia de algunos aspectos a dificultar a las familias su participación en el centro: el horario laboral de los padres y madres (entre algunas veces y a menudo; $\mathrm{M}=3.56$, $\mathrm{DT}=1.48$ ), percibir que nadie les ha invitado a participar (entre pocas y algunas veces, $\mathrm{M}=2.13$, DT=1.37), o tener que cuidar de hijos pequeños o de personas mayores (entre pocas y algunas veces, $\mathrm{M}=2.00$, DT $=1.34$ ). Con relación a las dificultades de los hijos cuando tienen bajas calificaciones, los padres y madres tienden a responder que se disgustan, les riñen o castigan entre algunas veces y a menudo, $(\mathrm{M}=3.29, \mathrm{DT}=1.35)$.

Además de los indicadores que ofrecen la media y la desviación típica, analizamos en este estudio indicadores porcentuales que resultan relevantes dado el tamaño de la muestra y el contenido y significado de las variables. Se ha estimado que una concentración de un $10 \%$ ( $\mathrm{n} \geq 88$ familias) o más en las opciones que señalan dificultades en la participación, merecen una especial mención. Tomando este criterio como referencia, los resultados indican la existencia de limitaciones en los siguientes indicadores (ordenados por magnitud porcentual al sumar las opciones de respuesta "a menudo" y "siempre"): 1) El horario de trabajo de los progenitores (59.5\%, 524 familias); 2) El horario del centro escolar (35.1\%, 309 familias); 3) percibir que no se les invita a participar (18.2\%, 160 familias); 4) tener que cuidar de hijos pequeños o de personas mayores $(17.7 \%, 156$ familias); 5) falta de interés en participar (10.5\%, 92 familias). 
Tabla 2. Resultados de los análisis descriptivos: frecuencias, porcentajes, medidas de tendencia central (media) y de variabilidad (desviación típica)

\begin{tabular}{|c|c|c|c|c|c|c|}
\hline \multirow[b]{2}{*}{$\begin{array}{l}\text { APOYO EMOCIONAL A LOS HIJOS ANTE DIFICULTADES ACADÉMICAS Y DIFICULTADES DE } \\
\text { LAS FAMILIAS PARA PARTICIPAR EN EL CENTRO ESCOLAR }\end{array}$} & \multirow[b]{2}{*}{$\mathbf{N}$} & \multirow[b]{2}{*}{ MEDIA } & \multirow[b]{2}{*}{ Sx } & \multicolumn{3}{|c|}{ PORCENTAJES AGRUPADOS (\%) } \\
\hline & & & & $\begin{array}{c}\% \mathrm{~N}+\mathrm{PV} \\
(\mathrm{N})\end{array}$ & $\% \operatorname{AV}(\mathrm{N})$ & $\% \mathrm{AM}+\mathrm{S}(\mathrm{N})$ \\
\hline $\begin{array}{l}\text { Cuando mi hijo/a me transmite problemas con el profesorado, le muestro mi apoyo } \\
\text { incondicional y critico al centro }\end{array}$ & 880 & 1.92 & 1.08 & $76.9 \%(677)$ & $12.5 \%(110)$ & $10.6 \%(93)$ \\
\hline $\begin{array}{l}\text { Intento justificar a mi hijo/a ante conductas que el profesorado califica como contrarias a } \\
\text { las normas de convivencia }\end{array}$ & 880 & 1.67 & 0.95 & $84.4 \%(743)$ & $9.1 \%(80)$ & $6.5 \%(50)$ \\
\hline $\begin{array}{l}\text { Si en las calificaciones que presenta mi hijo/a hay suspensos, le hago ver que me disgusta, } \\
\text { le riño o castigo }\end{array}$ & 880 & 3.29 & 1.35 & $28.3 \%(249)$ & $24.7 \%(217)$ & $47 \%(414)$ \\
\hline $\begin{array}{l}\text { Las dificultades que encuentro para participar en actividades del centro se deben al horario } \\
\text { de trabajo }\end{array}$ & 880 & 3.56 & 1.48 & 25.3 & $15.1 \%$ & 59 \\
\hline Las dificultades para participar en el centro escolar se deben al horario del colegio & 880 & 2.83 & 1.43 & $41.8 \%(368)$ & $23.1 \%(203)$ & $35.1 \%(309)$ \\
\hline $\begin{array}{l}\text { Las dificultades para participar en el centro escolar se deben al cuidado de mis hijo/as o de } \\
\text { personas mayores }\end{array}$ & 880 & 2.00 & 1.34 & $70.9 \%(624)$ & $11.4 \%(100)$ & $17.7 \%(156)$ \\
\hline Las dificultades para participar en el centro escolar se deben al transporte hasta el centro & 880 & 1 & 1.02 & $85 \%(67$ & $7.8 \%(69)$ & $7.2 \%(63)$ \\
\hline $\begin{array}{l}\text { Las dificultades para participar en el centro escolar se deben a que no siento interés en } \\
\text { participar }\end{array}$ & 880 & 1.84 & 1.19 & $72.3 \%(636)$ & $17.3 \%(152)$ & $10.5 \%(92)$ \\
\hline $\begin{array}{l}\text { Las dificultades para participar en el centro escolar se deben a que nadie me ha pedido que } \\
\text { participe }\end{array}$ & 880 & 2.13 & 1.37 & $66.4 \%(584)$ & $15.5 \%(136)$ & $18.2 \%(160)$ \\
\hline $\begin{array}{l}\text { Las dificultades para participar en el centro escolar se deben a que me siento a disgusto en } \\
\text { el centro }\end{array}$ & 880 & 1.42 & 0.90 & $87.7 \%(773)$ & $7.2 \%(63)$ & $5 \%(44)$ \\
\hline $\begin{array}{l}\text { Las dificultades para participar en el centro escolar se deben a que he tenido experiencias } \\
\text { negativas en este o en otro centro }\end{array}$ & 880 & 1.42 & 0.92 & $87.2 \%(767)$ & $7.7 \%(68)$ & $5.1 \%(45)$ \\
\hline
\end{tabular}

Nota: Variables: 1-Nunca (N), 2-Pocas veces (PV), 3- Algunas veces (AV), 4- A menudo (AM), 5- Siempre (S).

Fuente: Elaboración propia. 
Asimismo, sobre indicadores de Apoyo emocional a los hijos ante dificultades académicas se obtienen los siguientes resultados: 1) Cuando mi hijo tiene suspensos le hago ver que me disgusta, le riño o castigo ( $47 \%, 414$ familias); 2) Cuando mi hijo me transmite problemas con el profesorado, le muestro mi apoyo incondicional y critico al centro (10.6\%, 93 familias).

\subsection{Análisis comparativos entre grupos}

En esta segunda sección se presentan resultados procedentes de la comparación de grupos atendiendo a las variables sociodemográficas incluidas en el estudio: género de los progenitores, edad, nivel de estudios, situación laboral y número de hijos. El objetivo es identificar situaciones de diversidad familiar derivadas de estas variables, que incidan, por una parte, en la dificultad de los padres y madres para participar en los centros escolares, y, por otra, en su actitud de respuesta ante las dificultades académicas de sus hijos.

\subsubsection{Análisis comparativos en función del género de los padres y madres}

Respecto al género del progenitor se han calculado la t de Student estableciendo como diferencias entre medias el valor del padre menos el valor de la madre (padre-madre). Los resultados muestran que solo existen diferencias estadísticamente significativas al considerar las dificultades de participación en el centro asociadas al horario de trabajo: los padres tienen más dificultades que las madres $\left(\mathrm{t}\left(\mathrm{s80}_{\mathrm{O}}\right)=5,07 ; \mathrm{p}=0,000 ; \mathrm{d}=0.196\right)$. El tamaño del efecto, no obstante, es bajo.

En lo que respecta a la actitud de respuesta ante las dificultades académicas de los hijos no se han detectado diferencias significativas entre los padres y las madres.

\subsubsection{Análisis comparativos en función de la edad de los padres y madres}

La edad de los padres y las madres ejerce diferencias estadísticamente significativas entre los progenitores solo en la variable " $\mathrm{Si}$ en las calificaciones que presenta mi hijo hay suspensos, le hago ver que me disgusta, le riño o castigo" ( $F_{(2,878)}=7,33 ; p=0,001$ $\left.\mathrm{R}^{2}=.016\right)$. Los padres y madres más jóvenes -entre 30 y 40 años- presentan esta conducta con mayor frecuencia que quienes tienen una edad mediana, entre 41 y 51 años.

\subsubsection{Análisis comparativos en función del nivel de estudios de los padres y madres}

$\mathrm{Al}$ considerar el nivel educativo de los padres, se observan diferencias significativas en las siguientes variables: 1) "Cuando mi hijo me transmite problemas con el profesorado, le muestro mi apoyo incondicional y critico al centro” $\left(\mathrm{W}_{(2,878)}=3.84 ; \mathrm{p}=0.022 ; \mathrm{R}^{2}=.007\right)$ : los progenitores con nivel educativo primario tienen esta conducta con mayor frecuencia que aquellos con nivel educativo universitario $(\mathrm{p}=.001) ; 2)$ "Intento justificar a mi hijo ante conductas que el profesorado califica como contrarias a las normas de convivencia" ( $\mathrm{W}_{(2,}$ $\left.{ }_{878)}=10.19 ; \mathrm{p}=0.000 ; \mathrm{R}^{2}=.018\right)$ : al igual que sucedía en la variable anterior, los progenitores con nivel educativo primario justifican más a sus hijos que aquellos con nivel educativo universitario ( $\mathrm{p}=.000)$; 3) "Las dificultades que encuentro para participar en actividades del centro se deben al horario de trabajo" $\left(\mathrm{W}_{(2,878)}=4.05 ; \mathrm{p}=0.018 ; \mathrm{R}^{2}=.002\right)$ : los progenitores con nivel educativo primario tienen menos dificultades que aquellos con nivel educativo universitario ( $\mathrm{p}=.000)$ y 4 ) "Las dificultades para participar en el centro escolar se deben al horario del colegio" $\left(\mathrm{F}_{(2,878)}=4.25 ; \mathrm{p}=0.015 ; \mathrm{R}^{2}=.010\right)$ : en este caso, los padres con nivel educativo secundario tiene más dificultades que aquellos con nivel educativo universitario $(\mathrm{p}=.000)$.

3.2.4. Análisis comparativos en función de la situación laboral de los padres y madres 
Con relación a la situación laboral de los progenitores, los resultados muestran diferencias estadísticamente significativas en las siguientes variables: 1) "Si mi hijo suspende le hago ver que me disgusto, le riño o castigo" $\left(\mathrm{F}_{(1,879)}=3.49 ; \mathrm{p}=0.015 ; \mathrm{R}^{2}=.012\right)$ : los padres ocupados presentan este comportamiento con mayor frecuencia que los padres jubilados; 2) "Las dificultades que encuentro para participar en actividades del centro se deben al horario de trabajo" $\left.\left(\mathrm{W}_{(2,878)}=26.57 ; \mathrm{p}=0.000 ; \mathrm{R}^{2}=.002\right) ; 3\right)$ "Las dificultades para participar se deben al horario del centro" $\left(\mathrm{F}_{(1,879)}=4.66\right.$; $\mathrm{p}=0.015$; $\left.\mathrm{R}^{2}=.101\right)$; y 4) "Las dificultades para participar en el centro escolar se deben al cuidado de mis hijos pequeños o de personas mayores" $\left(\mathrm{W}_{(2,878)}=3.48 ; \mathrm{p}=0.023 ; \mathrm{R}^{2}=.016\right)$.

En las tres últimas variables citadas se encuentra, como cabría esperar, que los padres ocupados perciben más dificultades para participar en el centro escolar por su horario laboral y por el horario del centro que aquellos que desarrollan tareas domésticas. Por el contrario, quienes realizan tareas domésticas perciben más dificultades que quienes trabajan fuera del hogar en participar en el centro por tener que cuidar de niños pequeños o de personas mayores.

\subsubsection{Análisis comparativos en función del Número de Hijos de los padres y madres}

Respecto al número de hijos los resultados señalan diferencias estadísticamente significativas en las dificultades debidas al horario de trabajo $\left(\mathrm{W}_{(2,878)}=3.85 ; \mathrm{p}=0.022\right.$; $\left.\mathrm{R}^{2}=.009\right)$, al cuidado de los hijos o de personas mayores $\left(\mathrm{W}_{(2,878)}=17.36 ; \mathrm{p}=0.000\right.$; $\left.\mathrm{R}^{2}=.032\right)$, al transporte hasta el centro escolar $\left(\mathrm{W}_{(2,878)}=5.22 ; \mathrm{p}=0.006 ; \mathrm{R}^{2}=.0110\right)$, y a experiencias negativas en este $\mathrm{o}$ en otro centro escolar $\left(\mathrm{W}_{(2,878)}=5.64 ; \mathrm{p}=0.004 ; \mathrm{R}^{2}=.012\right)$. En estas variables los progenitores con dos hijos ( $\mathrm{p}=.000 ; \mathrm{p}=.016 ; \mathrm{p}=.023)$ y tres o más $(\mathrm{p}=.000 ; \mathrm{p}=.000 ; \mathrm{p}=.000 ; \mathrm{p}=.029 ; \mathrm{p}=.013)$ presentan mayores limitaciones que quienes tienen un solo hijo.

\section{Discusión}

La colaboración entre el centro escolar y la familia constituye una necesidad que es asumida por ambos contextos y por la sociedad en general, puesto que ninguno de estos dos agentes de socialización puede responder por sí mismo a todas las demandas que supone la educación de los niños y jóvenes en la sociedad actual (Christenson et al., 1992). Esta colaboración, que favorece su desarrollo integral y su rendimiento escolar, han de promoverla y desarrollarla los centros, tal como se plantea en la legislación educativa internacional y nacional (Ministerio Español de Educación, Cultura y Deporte., 2014). La finalidad es que el profesorado y las familias puedan compartir los mismos objetivos educativos para los niños y lleguen a conseguirlos a través de procesos de escucha, de comunicación y de toma de decisiones conjuntas (Santos y Lorenzo, 2009).

En las etapas de Educación Infantil y Primaria esta colaboración suele ser más frecuente que en etapas posteriores (Parra Martínez, García Sanz, Gomariz Vicente y Hernández Prados, 2014) y se vincula más directamente con los contenidos del curriculum, con el aprendizaje vivencial y cotidiano, y con la cercanía al entorno, entendiéndose, por ello, como un elemento clave para el éxito del proceso de enseñanza y aprendizaje y para contribuir a la formación plena de los niños y jóvenes. En la etapa de la Educación Secundaria Obligatoria, sin embargo, la relación entre las familias y el centro suele ser más esporádica y centrarse, sobre todo, en aspectos del rendimiento académico, en el 
control del fracaso y del absentismo escolar y en la orientación vocacional (Martínez González y Álvarez Blanco, 2005).

Numerosas investigaciones, así como los propios docentes y las familias, constatan que esta cooperación no es fácil de lograr y de consolidar (Van der Wolf y Everaert, 2005; García Bacete, 1996, 2006). La mayoría de las veces se reduce a la asistencia de los padres a las reuniones que organizan los centros para informar sobre aspectos curriculares y de funcionamiento, o bien a actividades extracurriculares, como eventos deportivos, culturales o festivos (Martínez González et al., 2000; Martínez González et al., 2005; Rivera y Milicic, 2006). Por ello, en este estudio se ha planteado identificar, por una parte, dificultades que tienen las familias para participar en el centro escolar de sus hijos y, por otra, su actitud hacia sus hijos y hacia el centro escolar cuando estos presentan dificultades en sus estudios.

Con respecto a este último aspecto, los resultados obtenidos indican que la mayoría de los padres y madres que han participado no tienden a justificar el comportamiento de sus hijos cuando no cumplen las normas de convivencia del centro; ni tampoco tienden a criticar al centro cuando sus hijos tienen problemas en él, como en ocasiones percibe el profesorado (Van der Wolf y Everaert, 2005), lo que indica que los padres y madres esperan que sus hijos tengan un comportamiento respetuoso dentro de él. En los escasos casos detectados en que los padres y madres actúan al contrario, sería de interés que se comunicaran con el profesorado para informarse directamente sobre los problemas que existen y tratar de llegar a encontrar conjuntamente una solución.

Atendiendo al género de los progenitores, se ha visto que de entre quienes justifican más a sus hijos, hay más madres que padres, mientras que sucede a la inversa entre quienes critican al centro. En ambos comportamientos parentales, se ha encontrado mayor frecuencia entre los padres y madres con estudios primarios que universitarios. Quizás los padres con mayor nivel de estudios y más experiencia vivida en el ámbito académico son más exigentes en esperar que sus hijos se adapten a las normas del centro y no justifican por ello su inadecuado comportamiento. Probablemente tienen expectativas de que sus hijos continúen más tiempo en el ámbito académico y, por ello, esperan que aprendan a comportarse adecuadamente en él.

Cuando los hijos suspenden, un poco más de la mitad de la muestra de padres y madres de este estudio suelen disgustarse, reñirles y castigarles, esperando un comportamiento más responsable en los aspectos académicos. Esto es más propio de las madres que de los padres que han participado en este estudio, y de los padres y madres que trabajan por comparación con aquellos que están jubilados, así como de quienes son más jóvenes -entre 30 y 40 años- por comparación con los de edades más maduras, de entre 41 y 51 años. Quizás los primeros se encuentran en un momento vital en el que se da más importancia al buen rendimiento académico para obtener en el futuro la acreditación necesaria para situarse en el mercado laboral. Por otra parte, es posible que estos padres y madres más jóvenes puedan tener más tendencia a resolver los problemas con sus hijos a través del castigo y la imposición que aquellos de edad más madura, que suelen tender a relativizar más la importancia de estos problemas.

Otros padres y madres no reaccionan de esta manera ante los suspensos, sino de un modo más sosegado y tranquilo, sin transmitir emociones negativas. Esta conducta parental puede resultar más relevante para reconducir de una forma más constructiva la implicación y responsabilidad de los hijos hacia sus estudios que cuando se hace de modo 
impositivo y culpabilizador. En este sentido, podría ser de interés orientar a los padres y madres en estrategias parentales para promover la motivación en sus hijos y para desarrollar con ellos procesos de negociación (Martínez González, 2009; Rodrigo et al., 2015).

Con respecto al segundo aspecto analizado en este estudio, es decir, las dificultades que tienen los padres y madres para participar físicamente en el centro escolar, es preciso considerar que éstas pueden estar asociadas a la propia institución educativa, a sus propios roles como padres y madres, al profesorado, a aspectos de tipo administrativo y organizativo, o a destrezas comunicativas del personal para hacer que los padres y madres se sientan a gusto en el centro, que de no utilizarse adecuadamente dificultan que éstos actúen como verdaderos espacios abiertos a la participación (Coulby, 2006).

En muchas ocasiones, se hace referencia desde los centros escolares al desinterés de las familias en implicarse en sus actividades (Walther, 2013), e incluso en el seguimiento escolar de sus hijos en casa, delegando su educación en el profesorado. Esta idea, sin embargo, no se constata en este estudio, donde solo se ha detectado un pequeño porcentaje de padres y madres que indican que no participan en los centros por desinterés. Y muy pocos señalan que una razón para no participar sean motivos emocionales derivados de relaciones difíciles con el profesorado, como encontrarse a disgusto o haber tenido experiencias negativas en el centro. Otras razones externas como problemas de transporte hasta el centro tampoco han resultado relevantes; no obstante, cabe decir que de entre quienes han indicado esta dificultad se encuentran más madres que padres, y aquellos que tienen tres o más hijos por comparación con los restantes. Quizás disponer de un coche propio que permita el desplazamiento al centro sea más difícil de conseguir para las madres y para las familias numerosas.

\section{Conclusiones}

Las familias que han participado en este estudio constatan que existen aspectos relevantes que limitan la posibilidad de implicarse en el centro.

Los horarios de trabajo, tanto de los propios padres y madres como del centro, constituyen un condicionante importante de la participación que resulta, en ocasiones, complejo de modificar. En muchas ocasiones, el profesorado intenta adaptar las actividades culturales de participación a los horarios disponibles de los padres y madres, así como los horarios de las entrevistas tutoriales. Aún así resulta complejo garantizar la asistencia. A esta dificultad se unen las responsabilidades familiares de muchos padres y madres de cuidar de hijos pequeños o de personas mayores en el hogar. De modo que, para una parte sustancial de padres y madres, se hace difícil conciliar su vida laboral, con la familiar y con la colaboración dentro del recinto escolar.

No obstante, estas limitaciones para la participación no se manifiestan de igual modo en todas las familias, poniendo de manifiesto la diversidad de tendencias de comportamiento.

Con respecto al género, se ha constatado en diversos estudios que tradicionalmente la madre está más implicada que el padre en la educación y en el centro escolar de sus hijos, y mantiene una relación más frecuente con el profesorado (Jares, 2006; García Bacete, 2006; Martínez-González, Álvarez Blanco y Pérez Herrero, 2013). Sin embargo, en los últimos años y como consecuencia de los cambios sociales y cultuales (incorporación de la mujer al mundo laboral, diversidad familiar, reducción de la natalidad, etc.), los padres se 
van involucrado en las acciones de crianza de sus hijos, favoreciendo la corresponsabilidad familiar (Maganto et al., 2010).

En relación con ello, los resultados obtenidos en este estudio no informan sobre diferencias sustanciales entre padres y madres en las dificultades que encuentran unos y otros para participar en el centro; tan solo se ha detectado que los padres tienen significativamente más dificultad que las madres al considerar el horario de trabajo. Otras áreas en las que se pueden observar ciertas dificultades mayores en las madres que en los padres, pero no de gran relevancia, tienen que ver con cuidar de hijos pequeños o de personas mayores, problemas de transporte hasta el centro o sentirse a disgusto en él. Sin embargo, a diferencia de las madres, los padres encuentran más barreras debidas al horario del centro. También se encuentran otras diferencias no sustanciales de índole emocional y motivacional como percibir que no les han invitado a participar, haber tenido experiencias negativas en algún centro, o simplemente que no sienten interés en participar, aunque esto se produzca en muy pocos casos.

La edad de los padres y madres no parece establecer diferencias en las dificultades que tienen para participar en el centro escolar. Sin embargo, al considerar su nivel educativo se ha visto que aquellos con estudios universitarios tienen más dificultades debido a su horario de trabajo o al horario del centro que los padres y madres con estudios primarios. Probablemente aquellos tienen circunstancias laborales más intensas y prolongadas que los padres y madres con nivel de estudio bajo.

Si se tiene en cuenta la situación laboral de los progenitores, los resultados indican, como cabría esperar, que los padres y madres que trabajan fuera del hogar perciben más dificultades para participar en el centro escolar por su horario laboral y por el horario del centro que aquellos que desarrollan tareas domésticas y que quienes están parados. Por el contrario, quienes realizan tareas domésticas perciben más dificultades en participar en el centro debido al cuidado de niños pequeños o de personas mayores que quienes trabajan fuera del hogar.

En relación con el número de hijos se ha constatado que cuantos más convivan en la familia, más responsabilidades tienen los padres y madres en casa y más dificultades encuentran para participar en el centro escolar. Estos padres indican que estas dificultades se asocian, sobre todo, a incompatibilidades con el horario de trabajo, o a tener que cuidar de niños pequeños o de personas mayores, o incluso a dificultades de transporte hasta el centro. Se observa también en este estudio que un número mayor de hijos puede incrementar la posibilidad de que los padres y madres tengan experiencias negativas en el centro, seguramente porque aumenta también la probabilidad de que con alguno de ellos se produzcan problemas de rendimiento o de comportamiento en el centro (RodríguezRuiz, 2012).

Para concluir, cabe decir que en este estudio se advierte que los padres y las madres participan diferencialmente en el centro escolar fundamentalmente en función del tiempo del que disponen teniendo en cuenta sus horarios laborales y las responsabilidades asociadas a la crianza y al cuidado de sus hijos o de personas mayores. Sus dificultades de participación no se deben tanto a falta de interés, como muchas veces se entiende, ni a factores motivacionales y emocionales relevantes, tales como haber tenido experiencias negativas en el centro. 
Dadas las dificultades de los progenitores para participar y la diversidad de circunstancias familiares que inciden en ello, se observa en la actualidad que los centros escolares recurren a recursos tecnológicos como las páginas web y el correo electrónico, o a las agendas escolares que los niños portan a sus padres y madres desde el centro y viceversa, para informarles de temas que ambos consideran de interés. Se advierte, en este sentido, que la comunicación entre padres y madres y profesorado tiende a ser cada vez más a distancia y no tanto presencial, con las ventajas y también las limitaciones que ello puede conllevar. Por tanto, cabe sugerir incrementar la calidad de la relación y comunicación entre las familias y los centros a través de los recursos tecnológicos, como complemento a las relaciones presenciales.

Los resultados de este estudio ponen de manifiesto también la necesidad de promover medidas para conciliar la vida personal, familiar y laboral en una sociedad que exige a los padres y madres responder a numerosas demandas en un espacio reducido de tiempo.

Entre las limitaciones a destacar en este estudio cabe señalar que los resultados cuantitativos obtenidos no se han contrastado con otros de naturaleza cualitativa procedentes, por ejemplo, de entrevistas en profundidad o de grupos de discusión, que permitieran ofrecer más detalles sobre las conclusiones mencionadas. Estos datos cualitativos son útiles para indagar sobre las argumentaciones, sentimientos $\mathrm{y}$ circunstancias que afectan a los padres y madres, y que pueden condicionar tanto su implicación en las actividades del centro como en el apoyo que muestran a sus hijos en sus tareas académicas.

Por otra parte, sería interesante analizar también la perspectiva del profesorado sobre las dificultades de los padres y madres para participar en el centro, y obtener así una visión más amplia sobre esta temática. Esta percepción del profesorado es relevante porque puede tener incidencia en la calidad de la relación que establezca el centro con las familias y en las decisiones que tomen éstas para participar.

Por tanto, conviene seguir profundizando en futuras investigaciones sobre estos aspectos para identificar con más detalle las dificultades de participación de las familias en los centros y promover líneas de intervención al respecto.

\section{Referencias}

Adams, K. S. y Christenson, S. L. (1998). Differences in parent and teacher trust levels: implications for creating collaborative family-school relationships. Special Services in the Schools, 14(2), 122. doi:10.1300/j008v14no1_01

Adams, K. S. y Christenson, S. L. (2000). Trust and the family-school relationship examination of parent-teacher differences in elementary and secondary grades. Journal of School Psychology, 38(5), 477-497. doi:10.1016/s0022-4405(00)00048-0

Anderson, K. J. y Minke, K. M. (2007). Parent involvement in education: toward an understanding of parents' decision making. The Journal of Educational Research, 100(13), 311-323. doi:10.3200/joer.100.5.311-323

Berends, M. (1995). Educational stratification and students' social bonding to school. British Journal of Sociology of Education, 16(3), 327-347. doi:10.1080/0142569950160304

Broc, M. A. (2006). Motivación y rendimiento académico en alumnos de la educación secundaria obligatoria y bachillerato LOGSE. Revista de Educación, 340, 379-414. 
Bronfenbrenner, U. (1979). The ecology of human development. Cambridge, MA: Harvard University Press.

Calero, J., Choi, A. y Waisgrais, S. (2010). Determinantes del riesgo de fracaso escolar en España: Una aproximación a través de un análisis logístico multinivel aplicado a PISA-2006. Revista de Educación, Número extraordinario, 225-256.

Castelli, S., Addimando, L., Pieri, M. y Pepe, A. (Eds.) (2011). Current issues in home-school-community partnership. Bolonia: I libri di Emil.

Castelli, S., Mendel, M. y Ravn, B. (Eds.) (2003). School, family, and community partnerships in a world of differences and changes. Gdansk: University of Gdansk.

Ceballos, E., Rodríguez, B., Correa, A. D., Rodríguez, J. y Correa, N. T. (2012). La voz del alumnado en el conflicto escolar. Revista de Educación, 359, 554-579.

Christenson, S. L., Rounds, T. y Franklin, M. J. (1992). Home-school collaboration: Effects, issues, and opportunities. En S. L. Christenson y J. C. Conoley (Eds.), Home-school collaboration: enhancing children's academic and social competence (pp. 19-52). Silver Spring, MD: National Association of School Psychologists.

Cohen, J. (1969). Statistical power analysis for the behavioral sciences. Nueva York: Academic Press.

Comisión Europea (2000). Informe europeo sobre la calidad de la educación escolar. Dieciséis indicadores de calidad. Bruselas: Dirección General de Educación y Cultura.

Coulby, D. (2006). Intercultural education: theory and practice. Intercultural Education, 17(39), 245257. doi:10.1080/14675980600840274

Cronbach, L. J. (1951). Coefficient alpha and the internal structure of test. Psychometrika, 16, 297333. doi:10.4135/9781412961288.n54

Davies, D. (1991). Schools reaching out: Family, school, and community partnerships for student Success. Phi Delta Kappan, 72(5), 376-380.

Deslandes, R. y Bertrand, R. (2005). Motivation of parent involvement in secondary-level schooling. The Journal of Educational Research, 98(3), 164-175. doi:10.3200/joer.98.3.164175

Domínguez Martínez, S. (2010). La Educación, cosa de dos: la escuela y la familia. Revista Digital para Profesionales de la Enseñanza, 8, 1-15.

Epstein, J. L. (2011). School, family and community partnerships. preparing educators and improving schools. Philadelphia, PE: Westview Press.

Epstein, J. L. y Sanders, M.G. (2006). Prospects for change: preparing educators for school, family, and community partnerships. Peabody Journal of Education, 81(2), 81-120. doi:10.1207/s15327930pje8102_5

Fernández, M., Mena, L. y Riviere, J. (2010). Desenganchados de la educación. Factores, procesos y dimensiones del fracaso y el abandono escolar en España. Barcelona: Fundación La CaixaColección Estudios Sociales.

García Bacete, F. J. (1996). La participación de las familias en la educación de los hijos. En R. A. Clemente y C. Hernández (Coords.), Contextos de desarrollo psicológico y educación (pp. 257281). Málaga: Aljibe.

García Bacete, F. J. (2006). Cómo son y cómo podrían ser las relaciones entre escuelas y familias en opinión del profesorado. Cultura y Educación, 18(3-4), 247-265. doi: $10.1174 / 113564006779173000$ 
García Bacete, F. J y Traver, J. A. (2010). Familias, centroes educativos y comunidad. En F. J. García Bacete, A. Vaque y C. Gomis (Coords.). Intervención y mediación familiar (pp. 205249). Castellón de la Plana: Universidad Jaume I.

Garreta Bochaca, J. (2008). La participación de las familias en la escuela públicas. Madrid: CEAPA.

Gimeno, J. (1997). La transición a la educación secundaria. Madrid: Morata.

Glasgow, N. A. y Whitney, P. J. (2009). What successful schools do to involve families. Londres: Sage.

Hart, B. y Risley, R. T. (1995). Meaningful differences in the everyday experience of young American children. Baltimore, MD: Paul H. Brookes.

Hernández Baeza, A., Muñiz Fernández, J. y García Cueto, E. (2000). Comportamiento del modelo de respuesta graduada en función del número de categorías de la escala. Psicothema, 12, 288291.

Hiatt-Michael, D. (2001). Promising practices for family involvement in school. Greenwich, CT: Information Age Publishing, Inc.

INE. (2014). Proyección de la población de España 2014-2064. Recuperado de www.ine.es/prensa/np870.pdf

Jaeggi, J. M., Osiek, F. y Favre, B. (2003). Familles, école et quartier. De la solitude au sens: échec ou réussite scolaire d'enfants de milieu populaire. Ginebra: Service de la Recherche en Education.

Lozano, A. (2003). Factores personales, familiares y académicos que afectan al fracaso escolar en la educación secundaria. Revista Electrónica de Investigación Psicoeducativa y Psicopedagógica, $1(1), 43-66$.

Maganto, J. M., Etxeberria, J. y Porcel, A. (2010). La corresponsabilidad entre los miembros de la familia, como factor de conciliación. Educatio Siglo XXI, 28(1), 69-84.

Martín, M. y Gairín, J. (2007). La participación de las familias en la educación: un tema por resolver. Bordón 59(1), 113-151.

Martínez González, R. A. (1994). Diagnóstico de necesidades en la cooperación entre familia y centro escolar. Boston, MA: Institute for Responsive Education.

Martínez González, R. A. (1996). Familia y educación. Fundamentos teóricos y metodológicos. Oviedo: Servicio de Publicaciones de la Universidad de Oviedo.

Martínez González, R. A. (2009). Programa-guía para el desarrollo de competencias emocionales, educativas y parentales. Madrid: Ministerio de Sanidad y Política Social.

Martínez González, R. A. y Álvarez Blanco, L. (2005). Fracaso y abandono escolar en educación secundaria obligatoria: implicación de la familia y los centros escolares. Aula Abierta, 85, 127-146.

Martínez González, R. A., Pereira, M., Rodríguez, B., Peña, A., Martínez, R., García González, ..., Casielles, B. (2000). Dinamización de las relaciones familia-centro escolar a través de la formación del profesorado en este campo de actuación. Revista Española de Orientación y Psicopedagogía, 11(19), 107-120.

Martínez González, R. A., Pérez Herrero, M. H. y Rodríguez Ruiz, B. (Eds.) (2005). Family-schoolcommunity partnership merging into social development. Oviedo: Editorial SM.

Martínez González, R. A., Rodríguez, B. y Gimeno, J. J. (2010). Áreas de cooperación entre los centros docentes y las familias. Educatio Siglo XXI, 28(1), 127-156.

Martínez González, R. A., Rodríguez, B. y Rodrigo, M. J. (2012). Fathers' and teachers' perception about their partnership. En D. Hiatt-Michael y H. Z. Ho (Eds.), Promising practices for fathers' 
involvement in their children's education (pp.79-93). Charlotte, NC: Information Age Publishing.

Martínez-González, R. A., Álvarez Blanco, L. y Pérez Herrero, M. H. (2013). Teenagers at-risk of dropping out of high school. parents' and teachers' views on family involvement. En B. Boufoy-Bastick (Ed.), The international handbook of cultures of educational policy: comparative international issues in policy-outcome relationships. Achievement with family \& community involvement (pp. 221-258). Strasbourg: Analytics.

Ministerio de Educación Cultura y Deporte. (2014). La participación de las familias en la educación escolar. Madrid: Secretaría General Técnica.

Oliva, A. y Parra, A. (2004). Contexto familiar y desarrollo psicológico durante la adolescencia. En E. Arranz (Ed.), Familia y desarrollo psicológico (pp. 96-123). Madrid: Pearson Educación.

Parra Martínez, J., García Sanz, M. P., Gomariz Vicente, M. A. y Hernández Prados, M. A. (2014). Perfiles de participación de las familias españolas en los centros educativos. En MECD, La participación de las familias en la educación escolar (pp. 127-148). Madrid: Secretaría General Técnica. Ministerio de Educación Cultura y Deporte.

Pepe. A. y Addimando, L. (2010). Testing the psychometric properties of the challenging parent standard questionnaire (CPSQ) in the italian educational context. International Journal about Parents in Education, 4(1), 49-64.

Pérez García, F. (Coord.) (2015). Servicios públicos, diferencias territoriales e igualdad de oportunidades. Bilbao: Fundación BBVA.

Phtiaka, H. y Symeonidou, S. (2007). Schools and families in partnerships: looking into the future. Nicosia: University of Cyprus.

Rivera, M. y Milicic, N. (2006). Alianza familia-escuela: percepciones, creencias, expectativas y aspiraciones de padres y profesores de enseñanza general básica. PSTKHE-Escuela de Psicología, 15(1), 119-135. doi:10.4067/s0718-22282006000100010

Rodrigo López, M. J., Máiquez Chavez, M. L., Martín Quintana, J. C., Byrne, S. y Rodríguez Ruiz, B. (2015). Manual práctico de parentalidad positiva. Madrid: Síntesis.

Rodrigo, M. J., García, M., Máiquez, M. L., Rodríguez, B. y Padrón, I. (2008). Estrategias y metas en la resolución de conflictos cotidianos entre adolescentes, padres y madres. Infancia y Aprendizaje, 3(3), 347-362.

Rodríguez-Ruiz, B. (2012). Resolución de conflictos y desarrollo positivo en la adolescencia: Efecto de la coherencia familia-escuela. (Tesis doctoral). Universidad de La Laguna, Santa Cruz de Tenerife, España.

Rodríguez-Ruiz, B. Rodrigo-López, M. J. y Martínez-González, R. A. (2015). Cross-contextual variability in parents' and school tutors' conflict resolution styles and positive development. The Journal of Educational Research, 108(6), 480-491. doi:10.1080/00220671.2014.905764

San Fabián, J. L. (1994). La participación. Cuadernos de Pedagogía, 222, $18-21$.

Santos, M. A. y Lorenzo, M. M. (2009). La participación de las familias inmigrantes en la escuela. Un estudio centrado en la procedencia. Revista de Educación, 350, 277-300. doi: $10.18172 /$ con. 643

Sinclair, M. F., Lam, S. F., Christenson, S. L. y Evelo, D. (1993). Action research in middle schools: anwatin and northeast middle schools. Equity and Choice, 10, 22-24.

Smit, F. y Driessen, G. (2005). Parent-school-community relations in a changing society: Bottlenecks, pitfalls and solutions. En R. A. Martínez González, M. H. Pérez Herrero y B. 
Rodríguez (Eds.), Family-school-community partnerships merging into social development (pp. 171-190). Oviedo: Editorial SM.

Van der Wolf, K. y Everaert, H. (2005). Challenging parents, teacher characteristics and teacher stress. En R. A. Martínez-González, M.H. Pérez-Herrero y B. Rodríguez-Ruiz (Eds.), Family-school-community partnerships merging into social development (pp. 233-253). Oviedo: Editorial SM.

Walther, A. (Coord.). (2013). The view from the principal's office. The opinions of principals from 8 european countries compared. Comparative analysis institutional. Frankfurt: University of Frankfurt.

\section{Breve CV de los autores}

\section{Beatriz Rodríguez-Ruiz}

Doctora en Psicología (Universidad de La Laguna), Licenciada en Pedagogía (Universidad de Oviedo) y Experta en Intervención y Mediación Familiar, es Profesora Asociada de Universidad en el Departamento de Ciencias de la Educación de la Universidad de Oviedo. Ha recibido el Premio Extraordinario de Doctorado por su Tesis Doctoral con Mención Internacional: "Resolución de conflictos y desarrollo positivo en la adolescencia: Efecto de la coherencia familia-escuela" (Universidad de La Laguna) y es Convenor de la European Educational Research Association en la Network on Communities, Families, and Schooling in Educational Research. Su labor de investigación está relacionada con el ámbito familiar y la relación centros escolares-familias, los procesos de formación de profesionales del ámbito de educación, servicios sociales, sanidad y justicia en Parentalidad Positiva, así como en la implementación de programas socioemocionales y educativos con familias y profesionales.

\section{Raquel-Amaya Martínez-González}

Catedrática de Universidad Acreditada (ANECA) y Profesora Titular de Universidad en el Departamento de Ciencias de la Educación de la Universidad de Oviedo (España). Coordina el Grupo de Investigación "Intervención Educativa en el ámbito Familiar, Escolar y Social” (IEFES) y desarrolla estudios y programas educativos con Familias y Profesionales del sector Educativo, Servicios Sociales, de la Salud y otros sectores afines. Ha recibido Premios Nacionales en España por sus trabajos sobre Familia y Educación.Ha presidido el European Research Network About Parents in Education (ERNAPE). Es miembro del International Network of Scholars on School-Family-Community Partnerships (INET), USA. Es Convenor de la European Educational Research Association en el Network on Communities, Families, and Schooling in Educational Research. Ha difundido sus resultados de investigación en libros, revistas y conferencias nacionales e internacionales.

\section{María José Rodrigo López}

Doctora en Psicología y catedrática de Psicología Evolutiva y de la Educación de la Universidad de La Laguna, España. Es directora del Máster oficial interuniversitario en Intervención y Mediación Familiar de la Universidad de La Laguna y la Universidad de 
Las Palmas de Gran Canarias. Autora de numerosas publicaciones internacionales y nacionales entre las que destacan los libros de Familia y Desarrollo Humano, Preservación Familiar y el Manual Práctico de Parentalidad Positiva. Es experta consultora del Ministerio de Sanidad, Asuntos Sociales e Igualdad en temas de Parentalidad Positiva y colabora con la Federación Española de Municipios y Provincias en la elaboración de materiales para la difusión de estos temas. 